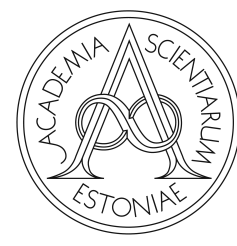

Proceedings of the Estonian Academy of Sciences,

2022, 71, 1, 55-64

https://doi.org/10.3176/proc.2022.1.05

Available online at www.eap.ee/proceedings

WAVELETS AND

FRACTIONAL

CALCULUS

\title{
Haar wavelet fractional derivative
}

\author{
Dedicated to Professor Ülo Lepik on his 100th birthday: he enlighted a century with his \\ outstanding life and brilliant work
}

\author{
Carlo Cattani
}

Engineering School, DEIM, University of “La Tuscia”, Via del Paradiso 47, 01100 Viterbo, Italy; cattani@unitus.it

Received 11 November 2021, accepted 27 December 2021, available online 4 February 2022

(C) 2022 Author. This is an Open Access article distributed under the terms and conditions of the Creative Commons Attribution 4.0 International License CC BY 4.0 (http://creativecommons.org/licenses/by/4.0).

\begin{abstract}
In this paper, the fundamental properties of fractional calculus are discussed with the aim of extending the definition of fractional operators by using wavelets. The Haar wavelet fractional operator is defined, in a more general form, independently on the kernel of the fractional integral.
\end{abstract}

Key words: wavelet theory, fractional calculus, Haar wavelet, operational matrix.

\section{INTRODUCTION}

In recent years, fractional calculus has been applied in several interesting topics from many different fields. This new approach, based on a suitable definition of fractional operators, has enabled to handle fundamental problems by showing unexpected results and new horizons in research (see, e.g., $[15,29,43]$ and references therein).

Unfortunately the definition of fractional derivative is not unique, and therefore, there is a deep controversial debate on the legitimacy of these operators. Moreover, the physical meaning of the fractional order parameter is still missing. As a consenquence, there is a wide choice of fractional operators with newly born candidates, and a deep quest for a physical interpretation of the solution to fractional differential problems. (see, e.g., $[13,28,29,40,43]$.

An interesting definition of fractional derivative, based on the sinc-function, was recently given in [42]. This function is very popular in signal analysis. Moreover, it is the fundamental basic function for the socalled Shannon wavelets [7-12]. In [42] the authors proposed the sinc-function as the kernel of the fractional operator, but since the sinc-function is the mother wavelet (for the Shannon family), this idea suggests us the possibility of considering a more general class of fractional operators, where the kernels are wavelets or (more generally) wavelet series. In this paper, such a class of wavelet fractional operators will be proposed and the corresponding theory discussed.

Wavelet theory has been a very rich and fast-evolving research topic. It has led to a large number of papers dealing with several issues from distant fields, giving rise to a wide distribution of different wavelet families. Among the many families of wavelets the most popular and smart family is the the so-called Haar 
wavelet family [27], which has been used by Lepik to solve several nonlinear differential problems by a smart method [22-25]. Many applications and models based on Haar wavelets have shown their flexibility and suitability to understand complex issues and to provide solutions to nonlinear problems as well [3,1820,30-33,37,39]. In [26], Lepik also solved a fractional integral equation by using Haar wavelet series for the integrating function. Proceeding from Lepik's original idea, a generalization is proposed below, where both the integrating function and the kernel can be represented as a wavelet series (in the most general case) or as a Haar wavelet series in the particular case of these special functions.

Wavelets enjoy many special properties such as their localization in time (or frequency) and the multiscale decomposition. In particular, the multiscale property enables to decompose the approximation space into separate scales [6,16], thus focusing on the main physical contribution at each scale. We will see that the fractional order parameter might have a suitable physical interpretation by the multi-scale approach, so that the interpolation factor could be explained as a zoom in and zoom out through the multi-scales of the differential problem.

The paper is organized as follows. Preliminary remarks on fractional operators are given in Section 2. Section 3 shows how to combine wavelets and fractional operators, and discusses the many possibilities offered by the wavelet series representation. Section 4 provides the basic properties of the Haar wavelet and the Haar wavelet fractional derivative is explicitly computed. Section 5 presents the conclusions.

\section{PRELIMINARY REMARKS ON FRACTIONAL CALCULUS}

The fractional derivative was conceived in the 18th century. It can be substantially derived as a generalization of the following.

Theorem 1 (Cauchy). Let $f(x) \in C_{0}$ be a continuous function on reals and $n>0$ a given integer, the Cauchy formula for the repeated integral is

$$
J_{a}^{-n} f(x) \stackrel{\text { def }}{=} \int_{a}^{x} \int_{a}^{t_{1}} \ldots \int_{a}^{t_{n-1}} f\left(t_{n}\right) d t_{n} \ldots d t_{2} d t_{1}=\frac{1}{(n-1) !} \int_{a}^{x}(x-t)^{n-1} f(t) d t .
$$

Proof. The proof can be easily obtained by induction, by showing that

$$
\frac{d}{d x} J_{a}^{-(n+1)} f(x)=n J_{a}^{-n} f(x), \quad \frac{d}{d x} J_{a}^{-1} f(x)=f(x) .
$$

In particular, it is

$$
\frac{d^{n}}{d x^{n}} J_{a}^{-n} f(x)=f(x)
$$

so that the $n$-th integer order derivative

$$
D^{n} \stackrel{\text { def }}{=} \frac{d^{n}}{d x^{n}}
$$

is the inverse operator of the repeated integral $J_{a}^{-n}$.

Let us assume that $f(x) \in C_{1}$ is a differentiable function so that from (2) we can easily get

$$
D^{n+1} J_{a}^{-n} f(x) \stackrel{\text { def }}{=} \frac{d}{d x}\left[\frac{d^{n}}{d x^{n}} J_{a}^{-n} f(x)\right]=f^{\prime}(x) .
$$

Let $\alpha$ be a rational number, and $n=1$. In order to proceed, we need to define the generalized repeated integral with a rational number. But unfortunately, we have a variety of integrals and, as a consequence, a variety of fractional operators. 


\subsection{Some of the most popular fractional derivatives}

In this section, some of the most popular definitions of fractional derivatives (see, e.g., [34,38]) are provided.

Let us start with the Riemann-Liouville integral and the corresponding derivative.

Definition 1. The Riemann-Liouville integral of fractional order $v \geq 0$ of a function $f(x)$ is defined as

$$
\left(J^{v} f\right)(t)=\left\{\begin{array}{l}
\frac{1}{\Gamma(v)} \int_{0}^{t}(t-\tau)^{v-1} f(\tau) d \tau, \quad v>0 \\
f(t), \quad v=0
\end{array}\right.
$$

For the Riemann-Liouville fractional operator $J^{\alpha}$ the following properties hold:

$$
\begin{array}{ll}
\text { (a) } J^{\alpha}\left(J^{\beta} f(t)\right) & =J^{\beta}\left(J^{\alpha} f(t)\right), \\
\text { (b) } J^{\alpha}\left(J^{\beta} f(t)\right) & =J^{\alpha+\beta} f(t), \\
(c) J^{\alpha} t^{v} & =\frac{\Gamma(v+1)}{\Gamma(\alpha+v+1)} t^{v+\alpha}, \quad \alpha, \beta \geq 0, v>-1, \\
\text { (d) } J^{v} e^{\lambda t} & =\frac{1}{v \Gamma(v)} e^{\lambda t} t^{v}, \quad v>0, \\
\text { (e) } J^{v} c & =\frac{c}{v \Gamma(v)} t^{v}, \quad v>0 .
\end{array}
$$

From this definition the corresponding derivative follows as shown below.

Definition 2. Riemann-Liouville fractional derivative of order $\alpha>0$ is defined as

$$
D_{R L}^{\alpha} f(t)=\frac{d^{n}}{d t^{n}} J^{n-\alpha} f(t), \quad n \in \mathbb{N}, n-1<\alpha \leq n
$$

The main problem with this derivative is the unvanishing value for a constant function. Therefore, the following was proposed by Caputo [38].

Let $f(x) \in C^{n}$ be an $n$-differentiable function, $\alpha$ a positive value, then the following holds.

Definition 3. The $\alpha$-order Caputo fractional derivative is defined as

$$
D_{C}^{\alpha} f(x)= \begin{cases}\frac{d^{n} f(x)}{d x^{n}}, & 0<\alpha \in \mathbb{N}, \\ \frac{1}{\Gamma(n-\alpha)} \int_{0}^{x} \frac{f^{(n)}(\tau)}{(x-\tau)^{\alpha-n+1}} d \tau, t>0, & 0 \leq n-1<\alpha<n,\end{cases}
$$

where $n$ is an integer, $x>0$, and $f \in C^{n}$. 
It can be easily shown that

$$
\begin{aligned}
& \text { (a) } J^{\alpha} D_{C}^{\beta} f(x)=f(x)-\sum_{k=0}^{n-1} f^{(k)}\left(0^{+}\right) \frac{x^{k}}{k !}, \\
& \begin{array}{ll}
\text { (b) } D_{C}^{\alpha} J^{\alpha} f(x)=f(x) . & \text { for } n \in \mathbb{N}_{0} \text { and } \alpha<n,
\end{array} \\
& \text { (c) } D_{C}^{\alpha} t^{n}= \begin{cases}0, & \text { otherwise, } \\
\frac{\Gamma(n+1)}{\Gamma(n-\alpha+1)} t^{n-\alpha}, & \\
\text { (d) } D_{C}^{\alpha} D_{C}^{\beta} f(x)=D_{C}^{\beta} D_{C}^{\alpha} f(x) .\end{cases}
\end{aligned}
$$

\subsection{The kernel of the fractional integral}

Riemann-Liouville (RL) and Caputo (C) derivatives are the most popular derivatives and they have been used in many applications (see, e.g., [2,5,11,12,14,17,21,35,36,40,41]). Nevertheless, they both suffer some drawbacks. In fact, the RL-derivative is unvanishing when $f(x)$ is constant. The Caputo derivative, instead, is defined on a singular kernel, which seems to be a problem. Therefore, many authors have tried to avoid these issues by defining some more flexible non-singular derivatives.

Indeed, the more general fractional derivative with a given kernel $K(x, \alpha)$, which generalizes the $\mathrm{C}$ derivative, is

$$
D^{\alpha} f(x)= \begin{cases}\frac{d^{n} f(x)}{d x^{n}}, & 0<\alpha \in \mathbb{N}, \\ \int_{0}^{x} f^{(n)}(\tau) K(x-\tau, \alpha) d \tau, t>0, & 0 \leq n-1<\alpha<n .\end{cases}
$$

The kernel should be defined in such a way that at least the two conditions

$$
\lim _{\alpha \rightarrow 0} K(x-\tau, \alpha)=1, \quad \quad \lim _{\alpha \rightarrow 1} K(x-\tau, \alpha)=\delta(x-\tau)
$$

hold true. Moreover, in order to be a non-singular kernel, it should be also

$$
\lim _{x \rightarrow \tau} K(x-\tau, \alpha) \neq 0, \quad \forall \alpha .
$$

Although there are several definitions of derivatives, they all depend on a kernel. In particular, it can be easily seen that the C-derivative [4], the Caputo-Fabrizio (CF) derivative [5] and the Atangana-Baleanu $(\mathrm{AB})$ derivative [1] are some special cases of (5) corresponding to the kernels, respectively:

$$
\begin{aligned}
\text { (C) } \quad K(x-\tau, \alpha) & =\frac{1}{\Gamma(n-\alpha)}(x-\tau)^{n-\alpha-1} \\
(C F) \quad K(x-\tau, \alpha) & =\frac{M(\alpha)}{1-\alpha} e^{-\frac{\alpha}{1-\alpha}(x-\tau)} \\
(A B) \quad K(x-\tau, \alpha) & =\frac{B(\alpha)}{1-\alpha} E_{\alpha}\left(-\frac{\alpha}{1-\alpha}(x-\tau)\right),
\end{aligned}
$$

where the Mittag-Leffler function is defined as

$$
E_{\alpha}(x) \stackrel{\text { def }}{=} \sum_{k=0}^{\infty} \frac{x^{\alpha k}}{\Gamma(\alpha k+1)} .
$$

It can be easily shown that the proposed kernels fulfill (6) while only (CF) and (AB) also fulfill the condition (7). 


\section{COMBINING WAVELETS AND FRACTIONAL OPERATORS}

The general structure of Caputo-based fractional derivative

$$
D_{C}^{\alpha} f(x)=\int_{0}^{x} f^{\prime}(\tau) K(x-\tau, \alpha) d \tau, \quad 0<\alpha<1
$$

is based on the kernel $K(x-\tau, \alpha)$, which is a positive function with decay to infinity (to ensure convergence), similar to the functions (8), to fulfill at least the conditions (6). The general structure of the RiemannLiouville first order derivative according to (4) is

$$
D_{R L}^{\alpha} f(x)=\frac{1}{\Gamma(1-\alpha)} \frac{d}{d x} \int_{0}^{x} f(\tau)(x-\tau)^{-\alpha} d \tau, \quad 0<\alpha \leq 1 .
$$

In some recent papers it has been proposed to assume for the kernel a power series expression, such as in the case of the Mittag-Leffler function (9) so that the modern fractional Caputo-like operators can be defined as

$$
D^{\alpha} f(x)=\int_{0}^{x} f^{\prime}(\tau) \sum_{i=0}^{\infty} a_{i}(x, \alpha) \tau^{i} d \tau
$$

analogously for the Riemann-Liouville type by expressing the kernel $(8)_{1}$ by a power series.

As a suitable generalization of (12), we can assume that either or both the function and the kernel could be expressed in terms of wavelet series. Thus, let $\psi_{j}(\tau)$ be a family of wavelets and we can have the following result.

Definition 4. The wavelet scale approximation of the classical fractional derivative is defined as

$$
D^{\alpha} f(x)=\int_{0}^{x} \sum_{j=0}^{\infty} b_{j} \psi_{j}(\tau) K(x-\tau, \alpha) d \tau
$$

However, by this definition, the wavelet representation of the integrand function $f(\tau)$ can be easily taken and, therefore, it is easy to have a numerical approximation of the resulting operator at different scales. Nevertheless, it does not give a better description of the fractional order parameter $\alpha$. Moreover, we can have the wavelet approximation within the so-called modern fractional approach by taking the kernel as a power series.

Definition 5. The wavelet scale approximation of the modern fractional derivative is defined as

$$
D^{\alpha} f(x)=\int_{0}^{x} \sum_{j=0}^{\infty} b_{j} \psi_{j}(\tau) \sum_{i=0}^{\infty} a_{i}(x, \alpha) \tau^{i} d \tau
$$

However, this operator can be seen as a hybrid fractional operator, which in any case has a wavelet approximation for the integrating function and a power approximation for the kernel.

A more general approach is to define a scale wavelet fractional operator, where the kernel is taken as a wavelet series. In this way we can single out the contribution of the kernel at each scale, so that the fractional parameter $\alpha$ can be seen as the contribution to the rate of change of a function depending on the scale. Thus, it has a more significant physical meaning than an abstract dependence on a pure fractional parameter $\alpha$ so that we can give the following definition.

Definition 6. The modern wavelet fractional derivative is defined as

$$
D^{\alpha} f(x)=\int_{0}^{x} f^{\prime}(\tau) \sum_{i=0}^{\infty} c_{i}(x, \alpha) \psi_{i}(\tau) d \tau,
$$


analogously for the Riemann-Liouville operator, by expressing $(8)_{1}$ as a wavelet series. In this approach the kernel, which is the fundamental function for this operator, is decomposed at different scales. It might be related to the fractional order $\alpha$ (as shown in the next section), thus giving to $\alpha$ and to the corresponding operator a scale dependence.

Also, in this case we can have a wavelet approximation of the integrating function and, as a result, the following holds.

Definition 7. The wavelet scale approximation of the modern wavelet fractional derivative is defined as

$$
D^{\alpha} f(x)=\int_{0}^{x} \sum_{j=0}^{\infty} b_{j} \psi_{j}(\tau) \sum_{i=0}^{\infty} c_{i}(x, \alpha) \psi_{i}(\tau) d \tau
$$

It should be noted that these definitions are given in the most general approach without fixing a particular integrating function and/or a kernel. It is not a definition of a new fractional operator, but merely a new perspective from where these operators can be seen and a means to give the fractional parameter a physical meaning (represented by the dependence of the kernel on the scale of the wavelet approximation). Thus, at the coarse scale we re-obtain the ordinary differential operator.

Although the product of two series might look a cumbersome task, we can give a simpler form to the above equation (15) by using the Cauchy product approximation

$$
D^{\alpha} f(x) \cong \int_{0}^{x} \sum_{j=0}^{N} b_{j}^{(f)} c_{N-j}^{(K)}(x, \alpha) \psi_{j}(\tau) \psi_{N-j}(\tau) d \tau .
$$

As a consequence, the following definition holds.

Definition 8. The $N$-scale approximation of the wavelet fractional derivative is

$$
D^{\alpha} f(x)=\sum_{j=0}^{N} b_{j}^{(f)} c_{N-j}^{(K)}(x, \alpha) \int_{0}^{x} \psi_{j}(\tau) \psi_{N-j}(\tau) d \tau
$$

and we can get the following result.

Definition 9. The wavelet fractional derivative is defined as the limit

$$
D^{\alpha} f(x)=\lim _{N \rightarrow \infty} \sum_{j=0}^{N} b_{j}^{(f)} c_{N-j}^{(K)}(x, \alpha) \int_{0}^{x} \psi_{j}(\tau) \psi_{N-j}(\tau) d \tau,
$$

where a fundamental role is played by the symmetric operational matrix

$$
P_{j, N-j}(x) \stackrel{\text { def }}{=} \int_{0}^{x} \psi_{j}(\tau) \psi_{N-j}(\tau) d \tau .
$$

In the next section we will explicitly compute this operational matrix for the Haar wavelet basis and the Riemann-Liouville kernel.

\section{HAAR WAVELETS}

Let us consider the Haar function

$$
h(\tau)= \begin{cases}1, & \tau \in[0,1 / 2) \\ -1, & \tau \in[1 / 2,1) \\ 0, & \tau \notin[0,1) .\end{cases}
$$


The compressed and translated instances of this function give rise to the Haar wavelets in the interval $[0,1]$ :

$$
h_{i}(\tau)= \begin{cases}1, & \tau \in[a, b) \\ -1, & \tau \in[b, c), \quad i=m+k+1, \\ 0, & \tau \notin[a, c)\end{cases}
$$

where $a=\frac{k}{m}, b=\frac{k+1 / 2}{m}, c=\frac{k+1}{m}, m=2^{j}, j=0,1, \ldots, N, k=0,1, \ldots, m-1$.

The corresponding integrals are

$$
\int_{0}^{x} h_{i}(\tau) d \tau= \begin{cases}x-a, & x \in[a, b) \\ (b-a)+(b-x), & x \in[b, c), \quad i=m+k+1, \\ 0, & x>c\end{cases}
$$

where $a=\frac{k}{m}, b=\frac{k+1 / 2}{m}, c=\frac{k+1}{m}, m=2^{j}, j=0,1, \ldots, N, k=0,1, \ldots, m-1$.

Moreover, in order to compute the operational matrix (18), we also need the following values

$$
\int_{0}^{x} h_{i}(\tau) h_{j}(\tau) d \tau= \begin{cases}2^{-(i+j) / 2}(x-a) \delta^{i j}, & x \in[a, b) \\ 2^{-(i+j) / 2}[(b-a)+(b-x)] \delta^{i j}, & x \in[b, c), \quad i=m+k+1, \\ 0, & x>c\end{cases}
$$

where $a=\frac{k}{m}, b=\frac{k+1 / 2}{m}, c=\frac{k+1}{m}, m=2^{j}, j=0,1, \ldots, N, k=0,1, \ldots, m-1$ and $\delta^{i j}$ is the Kronecher symbol. They can be easily obtained by using the definition (19) and a direct computation.

If we define the box function $h_{1}(\tau)$ as

$$
h_{1}(\tau)=\left\{\begin{array}{ll}
1, & \tau \in[0,1) \\
0, & \tau \notin[0,1)
\end{array},\right.
$$

then we can approximate a continuous function $u(x)$ in the interval $[0,1]$ by the Haar series

$$
\begin{gathered}
u(\tau)=\sum_{i=1}^{\infty} c_{i} h_{i}(\tau), \quad(i=m+k+1), \\
c_{i}=2^{j} \int_{0}^{1} u(\tau) h_{i}(\tau) d \tau
\end{gathered}
$$

where $m=2^{j}, j=0,1, \ldots, N, k=0,1, \ldots, m-1$.

The orthogonality property holds as

$$
\int_{0}^{1} h_{i}(\tau) h_{s}(\tau) d \tau=\left\{\begin{array}{ll}
2^{-j}, & i=s \\
0, & i \neq s
\end{array},\right.
$$

where $m=2^{j}, j=0,1, \ldots, N, k=0,1, \ldots, m-1$.

Shifted Haar wavelets are a family of Haar functions on the interval $[0, x]$ defined as

$$
h_{i}(\tau, x)= \begin{cases}1, & \tau \in\left[\frac{k}{m} x, \frac{2 k+1}{2 m} x\right) \\ -1, & \tau \in\left[\frac{2 k+1}{2 m} x, \frac{k+1}{m} x\right), \quad i=m+k+1, \\ 0, & \tau \notin\left[k x, \frac{k+1}{m} x\right)\end{cases}
$$

where $m=2^{j}, j=0,1, \ldots, J, k=0,1, \ldots, m-1, N=2^{J}$. 


\subsection{Fractional Haar derivative at the $N$-scale approximation}

In this section the Haar wavelet fractional derivative (17) can be explicitly defined. By taking into account (20) from (17),(18), we have

$$
D^{\alpha} f(x)=\lim _{N \rightarrow \infty} \sum_{j=0}^{N} b_{j}^{(f)} c_{N-j}^{(K)}(x, \alpha) \int_{0}^{x} h_{j}(\tau, x) h_{N-j}(\tau, x) d \tau .
$$

It means

$$
D^{\alpha} f(x)=\lim _{N \rightarrow \infty} \sum_{j=0}^{N} 2^{-N} b_{j}^{(f)} c_{N-j}^{(K)}(x, \alpha)(x-a) \delta^{j, N-j}
$$

and since $\delta^{j, N-j} \neq 0$ only when $j=N / 2$, we have

$$
D^{\alpha} f(x)=\lim _{N \rightarrow \infty} 2^{-N} b_{N / 2}^{(f)} c_{N / 2}^{(K)}(x, \alpha)(x-a),
$$

that is

$$
D^{\alpha} f(x)=2^{-N} b_{N / 2}^{(f)} \lim _{N \rightarrow \infty} c_{N / 2}^{(K)}(x, \alpha)(x-a),
$$

so that this limit will depend only on the wavelet coefficients of the kernel. It should be noted that (22) gives a very simple expression of the Haar fractional derivative thanks to the use of the Haar wavelet. In this case, we have a simple limit depending only on the Haar wavelet coefficients of the kernel. Therefore, some new perspectives in fractional calculus can be opened by grouping kernels according to their wavelet coefficients. The equation (22) also confirms that Haar wavelets are the most suitable tool to handle differential-integral problems as shown by Lepik in his seminal and fundamental papers [22-27], which remain a milestone in modern mathematics.

\section{CONCLUSIONS}

In this paper, the Haar wavelet fractional operator has been defined in order to have a more general form of a fractional operator which is independent on the chosen kernel. This definition, based on wavelets, combines the many advantages of both wavelets and fractional operators, thus opening new perspectives in the modern fractional calculus.

\section{ACKNOWLEDGEMENTS}

The author is very grateful to the Estonian Academy of Sciences for support. In particular, the publication costs of this article were fully covered by the Estonian Academy of Sciences.

\section{REFERENCES}

1. Atangana, A. and Baleanu, D. New fractional derivative with nonlocal and non-singular kernel: Theory and applications to heat transfer model. Therm. Sci., 2016, 20(2), 763-769.

2. Bagley, R. On the equivalence of the Riemann-Liouville and the Caputo fractional order derivatives in modeling of linear viscoelastic materials. Fract. Calc. Appl. Anal., 2010, 10(2), 123-126.

3. Bulut, A., Oruç Ö. and Esen, A. Numerical solutions of fractional system of partial differential equations by Haar wavelets. CMES-Computer Modeling in Engineering \& Sciences, 2015, 108(4), 263-284.

4. Caputo, M. Linear model of dissipation whose Q is almost frequency independent - II. Geophys. J. Int., 1967, 13(5), 529-539.

5. Caputo, M. and Fabrizio, M. A new definition of fractional derivative without singular kernel. Prog. Fract. Differ. Appl., 2015, 1(2), 73-85. 
6. Cattani, C. Harmonic wavelets towards solution of nonlinear PDE. Comput. Math. Appl., 2005, 50(8-9), 1191-1210.

7. Cattani, C. Connection coefficients of Shannon wavelets. Math. Model. Anal., 2006, 11(2), 117-132.

8. Cattani, C. Shannon wavelet analysis. Proceedings of the 7th International Conference on Computational Science - ICCS 2007. Lecture Notes in Computer Science, 4488, Part II, Beijing, China, May 27-30, 2007 (Shi, Y., van Albada, G. D., Dongarra, J. and Sloot, P. M. A., eds). Springer, Berlin, Heidelberg, 2007, 982-989.

9. Cattani, C. Shannon wavelets theory. Math. Probl. Eng., 2008, 2008, 164808.

10. Cattani, C. Shannon wavelets for the solution of integrodifferential equations. Math. Probl. Eng., 2010, 2010, 408418.

11. Cattani, C. Fractional calculus and Shannon wavelet. Math. Probl. Eng., 2021, 2012, 502812.

12. Cattani, C. Local fractional calculus on Shannon wavelet basis. In Fractional Dynamics (Cattani, C., Srivastava, H. and Yang, X. J., eds). De Gruyter, Krakow, 2015, ch. 1.

13. Cattani, C. and Guariglia, E. Fractional derivative of the Hurwitz $\zeta$-function and chaotic decay to zero. J. King Saud Univ. Sci., 2016, 28(1), 75-81.

14. Cattani, C., Srivastava, H. M. and Yang, X.-J. Fractional Dynamics. De Gruyter Open, Warsaw, 2016.

15. Dalir, M. and Bashour, M. Applications of fractional calculus. Appl. Math. Sci., 2010, 4(21-24), 1021-1032.

16. Daubechies, I. Ten lectures on wavelets. CBMS-NSF Regional Conf. Ser. in Appl. Math. SIAM, Philadelphia, PA, 1992.

17. de Oliveira, E. C. and Tenreiro Machado, J. A. A review of definitions for fractional derivatives and integrals. Math. Probl. Eng., 2014, 2014, 238459.

18. Esen, A., Bulut, F. and Oruç, Ö. A unified approach for the numerical solution of time fractional Burgers' type equations. Eur. Phys. J. Plus, 2016, 131, 116.

19. Hein, H. and Feklistova, L. Free vibrations of non-uniform and axially functionally graded beams using Haar wavelets. Eng. Struct., 2011, 33(12), 3696-3701.

20. Hein, H. and Feklistova, L. Computationally efficient delamination detection in composite beams using Haar wavelets. Mech. Syst. Signal Process., 2011, 25(6), 2257-2270.

21. Kamata, M. and Nakamula, A. Riemann-Liouville integrals of fractional order and extended KP hierarchy. J. Phys. A: Math. Gen., 2002, 35(45), 9657-9670.

22. Lepik, Ü. Numerical solution of differential equations using Haar wavelets. Math. Comput. Simul., 2005, 68(2), $127-143$.

23. Lepik, Ü. Haar wavelet method for nonlinear integro-differential equations. Appl. Math. Comput., 2006, 176(1), 324-333.

24. Lepik, Ü. Numerical solution of evolution equations by the Haar wavelet method. Appl. Math. Comput., 2007, 185(1), 695-704.

25. Lepik, Ü. Application of the Haar wavelet transform to solving integral and differential equations. Proc. Estonian Acad. Sci., 2007, 56(1), 28-46.

26. Lepik, Ü. Solving fractional integral equations by the Haar wavelet method. Appl. Math. Comput., 2009, $214(2), 468-478$.

27. Lepik, Ü. and Hein, H. Haar Wavelets: With Applications. Springer, Berlin, 2014.

28. Li, C., Dao, X. and Guo, P. Fractional derivatives in complex planes. Nonlinear Anal. Theory Methods Appl., 2009, 71(5-6), $1857-1869$.

29. Liu, K., Hu, R.-J., Cattani, C., Xie, G.-N., Yang, X.-J. and Zhao, Y. Local fractional Z-transforms with applications to signals on Cantor sets. Abstr. Appl. Anal., 2014, 2014, 638648.

30. Majak, J., Pohlak, M. and Eerme, M. Application of the Haar wavelet-based discretization technique to problems of orthotropic plates and shells. Mech. Compos. Mater., 2009, 45(6), 631-642.

31. Majak, J., Shvartsman, B. S., Kirs, M., Pohlak, M. and Herranen, H. Convergence theorem for the Haar wavelet based discretization method. Compos. Struct., 2015, 126, 227-232.

32. Majak, J., Shvartsman, B. S., Karjust, K., Mikola, M., Haavajõe, A. and Pohlak, M. On the accuracy of the Haar wavelet discretization method. Compos. B. Eng., 2015, 80, 321-327.

33. Majak, J., Ratas, M., Karjust, K. and Shvartsman, B. S. Higher order Haar wavelet method for solving differential equations. In Wavelet Theory (Modammady, S., ed.). IntechOpen, 2020.

34. Odibat, Z. M. and Shawagfeh, N. T. Generalized Taylor's formula. Appl. Math. Comput., 2007, 186(1), $286-293$.

35. Ortigueira, M. D. and Tenreiro Machado, J. A. What is a fractional derivative? J. Comput. Phys., 2015, $293,4-13$.

36. Ortigueira, M. D. and Tenreiro Machado, J. A. Which derivative? Fractal Fract., 2017, 1(1), 1-13.

37. Oruç, Ö., Esen, A. and Bulut, F. A Haar wavelet approximation for two-dimensional time fractional reaction-subdiffusion equation. Eng. Comput., 2019, 35(1), 75-86.

38. Podlubny, I. Fractional Differential Equations: An Introduction to Fractional Derivatives, Fractional Differential Equations, to Methods of Their Solution and Some of Their Applications, vol. 198. Academic Press, New York, NY, 1998.

39. Ratas, M., Salupere, A. and Majak, J. Solving nonlinear PDEs using the higher order Haar wavelet method on nonuniform and adaptive grids. Math. Model. Anal., 2021, 26(1), 147-169.

40. Wang, X. Fractional geometric calculus: toward a unified mathematical language for physics and engineering. In Proceedings of The Fifth Symposium on Fractional Differentiation and its Applications (FDA12), Hohai University, Nanjing, China, May 14-17, 2012.

41. Yang, X.-J., Baleanu, D. and Srivastava, H. M. Local Fractional Integral Transforms and Their Applications. Academic Press, New York, NY, 2015. 
42. Yang, X.-J., Gao, F., Terneiro Machado, J. A. and Baleanu, D. A new fractional derivative involving the normalized sinc function without singular kernel. 2017, arXiv:1701.05590.

43. Zhao, Y., Baleanu, D., Cattani, C., Cheng, D.-F. and Yang, X.-J. Maxwell's equations on Cantor sets: a local fractional approach. Adv. High Energy Phys., 2013, 2013, 686371.

\section{Murrulist järku tuletis Haari lainikute abil}

\section{Carlo Cattani}

Käesolevas artiklis käsitletakse murrulise diferentsiaal- ja integraalarvutuse põhiomadusi eesmärgiga laiendada murruliste operaatorite definitsiooni. Töös defineeritakse murrulist järku tuletis, kasutades Haari lainikuid piirväärtusena, mis sõltub tuuma Haari lainikute kordajatest. 